\title{
Integrating Behavioral Health in Primary Care Using Lean Workflow Analysis: A Case Study
}

\author{
Constance van Eeghen, DrPH, Benjamin Littenberg, MD, Melissa D. Holman, RHIA, \\ and Rodger Kessler, PhD
}

Background: Primary care offices are integrating behavioral health (BH) clinicians into their practices. Implementing such a change is complex, difficult, and time consuming. Lean workflow analysis may be an efficient, effective, and acceptable method for use during integration. The objectives of this study were to observe $\mathrm{BH}$ integration into primary care and to measure its impact.

Methods: This was a prospective, mixed-methods case study in a primary care practice that served 8,426 patients over a 17-month period, with 652 patients referred to $\mathrm{BH}$ services. Secondary measures included primary care visits resulting in BH referrals, referrals resulting in scheduled appointments, time from referral to the scheduled appointment, and time from the referral to the first visit. Providers and staff were surveyed on the Lean method.

Results: Referrals increased from 23 to 37 per 1000 visits $(P<.001)$. Referrals resulted in more scheduled $(60 \%$ to $74 \% ; P<.001)$ and arrived visits $(44 \%$ to $53 \% ; P=.025)$. Time from referral to the first scheduled visit decreased (hazard ratio, 1.60; 95\% confidence interval, 1.37-1.88) as did time to first arrived visit (hazard ratio, 1.36; 95\% confidence interval, 1.14-1.62). Survey responses and comments were positive.

Conclusions: This pilot integration of BH showed significant improvements in treatment initiation and other measures. Strengths of Lean analysis included workflow improvement, system perspective, and project success. Further evaluation is indicated. (J Am Board Fam Med 2016;29:385-393.)

Keywords: Behavioral Medicine; Delivery of Health Care, Integrated; Primary Health Care; Quality Improvement

Primary care offices are struggling to meet the behavioral health $(\mathrm{BH})$ needs of their patients. Forty percent of primary care patients need behavioral health services, ${ }^{1}$ and $43 \%$ to $60 \%$ of those patients are treated solely in primary medicine..$^{2-4}$ Of those referred to specialty mental health services by their primary care providers, $50 \%$ to $90 \%$ fail to receive services. ${ }^{5}$ A survey of 6600 primary

This article was externally peer reviewed.

Submitted 3 June 2015; revised 11 December 2015; accepted 15 December 2015.

From the Departments of General Internal Medicine Research (CvE, BL) and Family Medicine (RK), and the Center for Clinical and Translational Science (CvE, BL, RK), University of Vermont, Burlington; and the James M. Jeffords Institute for Quality \& Operational Effectiveness, Measurement Group, University of Vermont Medical Center, Burlington (MDH).

Funding: none.

Conflict of interest: none declared.

Corresponding author: Constance van Eeghen, DrPH, University of Vermont College of Medicine, 89 Beaumont Ave, Burlington, VT 05405 (E-mail: cvaneegh@med.uvm.edu). care physicians found that appropriate mental health services are the most difficult subspecialties to access. ${ }^{6}$ Most external referrals for specialty mental health and substance abuse services never generate an appointment; most patients who make an appointment never initiate care. ${ }^{7}$ A strategy to meet these needs has emerged over the past 20 years in the form of $\mathrm{BH}$ services integrated with and co-located in primary care practices. ${ }^{8}$

Integration of $\mathrm{BH}$ is effective in improving medical outcomes for patients with acute or chronic diseases as well as responding to mental health and substance use issues that are generally untreated or inadequately treated in primary care. ${ }^{9}$ However, a recent survey of all patient-centered medical homes certified by the National Committee for Quality Assurance found that $<40 \%$ have any mental health/substance abuse/BH clinicians as part of their practices. When available, those clinicians are generally not integrated into practice flow. ${ }^{9} \mathrm{Al}-$ though the operational and clinical components 


\begin{tabular}{ll}
\hline Clinical & Full-time, on-site primary care behavioral health clinician $\left(1\right.$ per 7,500 patients $\left.{ }^{11}\right)$ \\
- Clinician availability for personal, face-to-face introductions ("warm handoffs") and consultations \\
- Brief, evidence-supported treatment interventions; other clinical care responsibilities \\
- Intensive training of primary care behavioral health clinicians using standardized protocols for a broad range \\
of psychological and medical problems amenable to behavioral health treatment \\
- Population (panel)-based care using measurement-based, stepped treatment and other resources \\
- Screening for mental health, substance abuse, and health behavior issues and provider decision support \\
- - Reamlessly integrated into patient flow \\
- Training providers and staff in behavioral care procedures \\
- Appointment frequency and interval of behavioral health clinician consistent with primary care \\
- Shared, transparent EHR with 2-way notes and access to information \\
- Care management coordination of referrals and information with specialty care as needed \\
- Brief interventions, which are lower-cost services, provided over shorter episodes of care \\
- Coordination of services and finances to optimize sustainability \\
- Negotiation of appropriate reimbursement \\
- Regular reports of performance, RVUs, and financial data \\
Finance
\end{tabular}

Data from Hunter and Goodie. ${ }^{10}$

EHR, electronic health record; RVU, relative value unit.

needed to integrate $\mathrm{BH}$ care are well documented ${ }^{10}$ (Table 1), lack of knowledge about how to implement BH services into primary care is a major obstacle. ${ }^{7}$ Implementation must address several key issues, including how deeply the $\mathrm{BH}$ clinician is involved in the practice and the processes of care, strategies for organizational and financial barriers, and opportunities to use health information technology. $^{12,13}$

Lean (also known as Lean Manufacturing ${ }^{14}$ or Lean Thinking ${ }^{15}$ ) uses a tightly structured approach to analyze workflow and is known to produce sustained, effective change in large health care organizations. ${ }^{16,17}$ Lean is conducted by a frontline team representing different work functions. The team produces a systems diagram that identifies problems, analyzes root causes, and produces agreement on redesigned office systems and processes. ${ }^{14}$ Team members collaborate to develop a report and implementation plan, using a prespecified format that incorporates patient and practice data. The team is responsible for producing a redesigned systems diagram, along with planned measures and expected countermeasures for anticipated problems. The team develops strategies for adapting to organizational culture and completes an action plan, with defined deliverables and dates. The Lean approach was modified for use in a primary care office practices, using a relatively brief period of time ( 8 hours) for team meetings that were organized around provider and staff scheduling constraints. ${ }^{18}$

Earlier efforts to integrate $\mathrm{BH}$ in primary care ${ }^{19}$ suggested the need for a systematic approach to implementation. Therefore we sought to observe the process of integration into primary care and measure its impact on referrals, treatment initiation rate, efficiency, and acceptance.

\section{Background}

Innovations in health care should fit the local context of each unique organization. ${ }^{20}$ This case study example of integration took place in an academic internal medicine practice in northern Vermont. In 2010 to 2011 the practice provided 21,200 primary care patient visits to cover 7500 patients who were served by 12 attending providers (totaling 6 fulltime equivalents [FTEs]), 36 residents (9 FTEs), and 42 staff (12 clinical and 30 nonclinical; 27 FTEs). The practice provided care 6 days/week, 47 hours/week, for both walk-ins and scheduled visits.

The practice served adults. Among patients, $80 \%$ were 19 to 64 years old, $19 \%$ were aged $\geq 65$ years, and $1 \%$ was $\leq 18$ years old. Most $(63 \%)$ were covered by commercial insurance, with another $22 \%$ covered by Medicare, $12 \%$ by Medicaid, and $3 \%$ by self-paid coverage, workers' compensation, or other public agencies. The top 8 diagnoses/ conditions among these patients were hyperten- 
sion, hyperlipidemia, back pain, depression, diabetes, anxiety, cough, and arthritis.

The practice had no integrated case or care managers, clinical pharmacists, or psychiatrists. Adjacent to the practice, a master's-level psychologist provided long-term psychotherapy for mental health issues only. The first integrated $\mathrm{BH}$ clinician was introduced to the practice immediately after the completion of this integration project, working full time (40 hours/week) from start up.

All clinicians in the practice were paid on a salary basis; most were assigned to $\geq 50 \%$ of clinic time and demonstrated a high degree of commitment to the success of the organization. Although no financial incentives were provided for improving care quality or exploring innovations, providers demonstrated a long-term commitment to quality improvement projects in the practice. ${ }^{18}$ All clinicians were expected to generate revenues above their salary expense. The integration of primary care was expected to improve care quality by increasing access to $\mathrm{BH}$ services and decreasing wait times for primary care providers, while also improving the practice's finances.

Recent regulatory and reimbursement changes in Vermont also played a role in this study. Before 2010, state and national leaders, including one of the authors (RK), began to explore integrated care as a new model for behavioral and primary care services through special task forces on workforce, reimbursement, and population-based service needs. State policymakers and leading local health insurers started to support changes in traditional $\mathrm{BH}$ programs at this time, modestly supporting $\mathrm{BH}$ interventions in adult primary care, obstetrics, neurology, and gastroenterology. 7,21,22 These changes allowed primary care services to bill directly for $\mathrm{BH}$ services using traditional psychotherapy codes and reduced prior approval requirements for these services. While an innovation at the time, such reimbursement procedures using standard codes are now common.

\section{Methods}

\section{Study Design}

This prospective, mixed methods case study observed a single primary care practice as it used the Lean approach to integrate a new $\mathrm{BH}$ services.

\section{The Intervention}

The model of integration of $\mathrm{BH}$ services suggests placing inside the clinic one full-time $\mathrm{BH}$ clinician (BHC) for every 7,500 patients ${ }^{11}$; this BHC works alongside primary care providers. The $\mathrm{BHC}$, a master's-level provider or a $\mathrm{PhD}$ psychologist, receives referrals for patients with specific BH needs and, when possible, meets them during the visit at which the referral is made (a "warm handoff" ${ }^{21}$ ). The BHC consults directly with the provider on coexisting medical conditions and provides a bridge to community mental health specialists. After conducting an assessment, the BHC may hand off the care to other specialists or may fully engage the patient in treatment for a defined period of time. The BHC documents patient care in the electronic health record (EHR), alongside other clinicians' notes. Office systems support referral, scheduling, documentation, consults with providers, team education, and billing. The BHC's scope of practice includes cognitive behavioral therapy and other evidence-based behavioral interventions for depression, anxiety, insomnia, substance abuse, smoking cessation, and other nonpsychotic mental health and behavioral disorders. It also supports the diet, exercise, medication adherence, stress, and behavioral aspects of medical conditions such as diabetes, lung disease, heart disease, and asthma. The BHC can also provide acute stabilization and triaging to specialty mental health services.

A 7-member quality improvement (QI) team of physicians, midlevel providers, nurses, and office staff studied the current workflow from a patient's first telephone call requesting a primary care appointment for an unstated $\mathrm{BH}$ need to a completed $\mathrm{BH}$ referral-that is, the workflow without internal $\mathrm{BH}$ services available. The team, supported by a QI facilitator, created a systems diagram of this process, identifying problems with the current process and changes needed to integrate the BHC into the future patient care process. ${ }^{23}$ This workflow analysis was accomplished in eight 1-hour segments scheduled to accommodate members' work schedules. The process was based on structured analysis, the requirement that all team members individually draw their own analytic diagrams and build on them from one meeting to the next, and supportive clinical and administrative leadership that championed the process with the team and the rest of the practice. 
The team collectively redesigned its office system over the course of 8 meetings to include:

1. Direct scheduling for an appointment with the BHC based on triage screening by a nurse (without a physician referral)

2. Immediate face-to-face introduction of the BHC when a primary care provider generates a mental health referral (a "warm handoff")

3. Creation of an appointment with the BHC on the day that the referral is made

4. Preauthorization of insurance coverage for $\mathrm{BH}$ services by front office staff

5. Expedited scheduling of external mental health visits

6. Coordination and follow-up communication by the BHC with external mental health specialists

7. Provision of acute stabilization $\mathrm{BH}$ services by the BHC when patients have to wait for mental health specialist services

The Lean approach required the team to identify the needs of patients, clinicians, and nonclinicians. The team members actively facilitated these recommendations by working with colleagues and managers inside the clinic and across the institution in monthly meetings and other communication forums.

\section{Implementation}

At the end of 8 hours of team meetings, implementation moved forward through a series of faculty, resident, and staff meetings, each focused on engaging all members of the practice in a successful outcome. For example, residents received formal training in which they met regularly with the BHC in educational sessions and case presentations. Attending physicians worked with the BHC to develop a "playbook," or written protocols, for 2 specific medical conditions (attention deficit hyperactivity disorder and chronic pain coupled with opioid therapy) to guide interactions with the BHC. The BHC, in turn, worked with medical providers to keep them aware of patients' progress between their primary care visits and to support their understanding and use of the service. This iterative process of learning and fine-tuning continued through the implementation stage.

The BHC served patients with medical needs (eg, smoking, headache, back pain, sleep disturbance) as well as those needing screening and re- ferral for substance abuse and brief interventions for mental health issues resulting in referral to community providers. In the relatively few cases for which referral sources were not immediately available, the BHC provided acute care services beyond the 4 to 6 visits anticipated for most patients.

\section{Data Collection and Review}

An EHR provided patient-specific data about referrals made during primary care appointments for the 12 months before and the 5 months after BHC integration. The data extraction process sought to include all patients who were seen by a primary care physician in the target clinic during the study periods; all referrals for ambulatory $\mathrm{BH}$, mental health, or substance abuse services; and all appointments for such services that followed the identified referrals for at least 2 years after the primary care visit. Referrals that were not followed by specialty appointments within the academic medical center are represented in the data analysis as patients with no treatment initiated, although some of these may have been treated by community mental health specialists. Conversely, some specialty referrals may not appear in the EHR at all, especially those during the period before the intervention, when robust office systems were not in place.

The primary outcome was treatment initiation rate, defined as the proportion of referrals resulting in ambulatory $\mathrm{BH}$ visits. The EHR was also used to measure the proportion of primary care visits resulting in $\mathrm{BH}$ referrals, the proportion of $\mathrm{BH}$ referrals resulting in scheduled appointments, days from referral order date to scheduled $\mathrm{BH}$ appointment date, and the days from referral order date to the first actual BH visit. Provider and staff surveys were conducted before and after the intervention to measure perceptions of the QI method.

All practice members were verbally informed of this research study, and 7 individuals were invited to join the team based on role and formal or informal leadership within the practice, an opportunity they were free to accept or decline. The Committees on Human Research at the University of Vermont reviewed and approved the study protocol.

Pre- and postintervention surveys were developed to collect from all practice members confidential provider and staff opinions (except residents because of timing) on the degree to which the Lean process was acceptable, effective at changing office processes, and effective at changing the clinic's out- 
Table 2. Responding Practice Member Characteristics, Including Providers and Staff Active in the Practice in the Periods Before (September-October 2010) and After the Intervention (April-May 2011)*

\begin{tabular}{lccc}
\hline & \multicolumn{3}{c}{ Respondents } \\
\cline { 2 - 3 } Characteristics & $\begin{array}{c}\text { Before the Intervention } \\
(\mathrm{n}=17)\end{array}$ & $\begin{array}{c}\text { After the Intervention } \\
(\mathrm{n}=14)\end{array}$ & $P$ Value \\
\hline Age (years), mean (SD) & $45(11)$ & $51(7)$ & .10 \\
Female sex, $\mathrm{n}(\%)$ & $14(82)$ & $10(59)$ & .99 \\
Providers, n (\%) & $5(29)$ & $11(8)$ & .45 \\
Time at the practice (years), mean (SD) & $10(7)$ & .74 \\
\hline
\end{tabular}

${ }^{*}$ Response rates in both periods were $40 \%$.

$\mathrm{SD}$, standard deviation.

come (eg, more successful BH referrals). We used a model of technology acceptance to develop questions regarding the Lean QI method. ${ }^{24}$ The face validity of questions on workflow and clinical effectiveness was tested in preliminary trials of the survey. Survey scores were constructed on 7-point Likert scales. The preintervention survey was administered 6 months before BHC integration; the postintervention survey was administered 2 months after BHC integration.

The results and conclusions of this study were presented to the participating providers and staff for confirmation and comments 10 months after BHC integration. Feedback was collected both orally and in writing on anonymous feedback forms. This feedback was used to re-review and confirm or modify conclusions.

\section{Statistical Analysis}

We used STATA 13.1 (Stata Corp., College Station, TX) for data management and descriptive statistics. These included comparisons across 2 time periods (before and after the intervention) using the Wilcoxon rank sum test for ordinal data, Fisher exact test for categorical data, and the log rank test for time-to-event data. All analyses were 2-tailed, with $P<.05$ required for statistical significance. Because 54 of the 652 referred patients $(8 \%)$ received $\mathrm{BH}$ referrals in both periods, the analyses were repeated without these patients.

\section{Results}

\section{Practice Member Characteristics}

Twelve providers and 30 staff were present in the practice at the start of the integration project, of whom 17 (40\%) responded to the study survey. Eight months later, after integration had been completed, 12 providers and 23 staff were present, of whom $14(40 \%)$ responded (Table 2). There were no significant differences between these time periods in respondent age, sex, years employed in the practice, or proportion of provider respondents relative to staff respondents.

\section{Patient Characteristics}

The practice treated 7515 unique patients in the period (12 months) before the intervention and 4962 in the period (5 months) after the intervention. Of these patients, 4,051 appeared in both time periods, for a net total of 8,426 patients. A total of 401 patients received $\mathrm{BH}$ referrals before intervention and 305 after intervention; 54 patients received referrals in both time periods, for a net total of 652 patients (Table 3). Patients referred to $\mathrm{BH}$ services were more likely to be depressed than the general primary care population (78\% vs $38 \%$ ), more anxious ( $62 \%$ vs $28 \%)$, and visited the primary care practice more often (6.8 vs 2.9 visits/patient/year). As expected, these chronically ill, working-age adults were younger than the primary care population overall (45.6 vs 50.4 years) and had lower rates of commercial insurance ( $44 \%$ vs $63 \%$ ). In all other respects, the 2 populations were alike.

\section{Patient Outcomes}

The practice generated $480 \mathrm{BH}$ referrals during 21,219 primary care visits before the intervention and 342 referrals during 9,180 visits after the intervention (Table 4). The referral rate increased from 23 referrals per 1000 primary care visits to 37 $(P<.001)$, and the rate at which referrals resulted in scheduled mental health appointments increased from $60 \%$ to $74 \%(P<.001)$. The rate of treatment initiation (scheduled appointments that pa- 
Table 3. Patient Characteristics for All Patients Presenting for Primary Care Visits and for Those Referred to Behavioral Health Services over 17 Months (February 2010 to June 2011)

\begin{tabular}{lcc}
\hline Characteristics & $\begin{array}{c}\text { Patients Presenting for Primary } \\
\text { Care Visits }(\mathrm{n}=8426)\end{array}$ & $\begin{array}{c}\text { Patients Referred to Behavioral } \\
\text { Health Services }(\mathrm{n}=652)\end{array}$ \\
\hline Patient demographics & & $45.2(16.6)$ \\
$\quad$ Age (years), mean (SD) & $50.5(16.9)$ & $363(56)$ \\
Female sex & $4,753(56)$ & $589(90)$ \\
White race & $7,709(91)$ & $284(44)$ \\
Commercial insurance & $5,273(63)$ & $617(95)$ \\
Vermont resident & $7,977(95)$ & $533(82)$ \\
Chittenden County resident & $6,796(81)$ & $512(79)$ \\
Behavioral issues & & $403(62)$ \\
Depression & $3,189(38)$ & $279(43)$ \\
Anxiety & $2,390(28)$ & $106(16)$ \\
Medical comorbidities & & $261(40)$ \\
Hypertension & $3,450(41)$ & $173(27)$ \\
Diabetes & $1,172(14)$ & $5.2(4.3)$ \\
Hyperlipidemia & $3,731(44)$ & $1,979(23)$ \\
Arthritis & $2.9(2.5)$ & \\
Total PCP visits per patient per year, mean (SD) & & \\
\hline
\end{tabular}

Data are $\mathrm{n}(\%)$ unless otherwise indicated.

PCP, primary care physician; SD, standard deviation.

tients kept) increased from $44 \%$ to $53 \%(P=.025)$. There were 54 duplicate patients in each period whose experiences cannot be considered independent of each other. When the analysis was repeated without these patients, referral scheduling increased from $59 \%$ to $73 \%(P=.001)$ and treatment initiation increased from $44 \%$ to $54 \%(P=.014)$. We retained the duplicate patients for the remainder of the analysis.

In unadjusted Cox proportional hazards analysis, time to scheduling was significantly lower after the intervention than before (hazard ratio, 1.60; 95\% confidence interval, 1.37-1.88). This relationship was essentially unchanged when using a multivariate analysis to correct for age, sex, insurance, place of residence, race, and medical problems (hazard ratio, 1.68; 95\% confidence interval, 1.43-1.98). The intervention was also significantly associated with time to initiation of therapy (hazard ratio, 1.36; $95 \%$ confidence interval, 1.14-1.62). Again, this relationship was virtually unchanged after correcting for the potential confounders described

Table 4. Results of Behavioral Integration Project for All Patients Presenting for Primary Care and Those Referred to Behavioral Health Services Before (February 2010-January 2011) and After the Intervention (February 2011June 2011)

\begin{tabular}{lccc}
\hline Measures & $\begin{array}{c}\text { Before the } \\
\text { Intervention }\end{array}$ & $\begin{array}{c}\text { After the } \\
\text { Intervention }\end{array}$ & $P$ \\
\hline Duration (months) & 12 & 9,180 \\
Primary care visits (n) & 21,219 & 342 \\
Referrals for ambulatory MH/BH (n) & 480 & 37 & $<$ \\
Referrals per 1000 primary care visits (n) & 23 & 243 & 74 \\
BH visits scheduled (n) & 278 & 173 & $<.001$ \\
Scheduling rate (\%) & 60 & 53 & .03 \\
Initial BH visits (n) & 205 & 44 & \\
Treatment initiation rate (\%) & & & \\
\hline
\end{tabular}

MH/BH, mental health/behavioral health. 
Table 5. Fractions of Patients Scheduled and Arrived for Behavioral Care for All Patients Referred to Behavioral Health Services Before (February 2010-January 2011) and After the Intervention (February 2011-June 2011)

\begin{tabular}{lccccc}
\hline & \multicolumn{2}{c}{ Fraction of Patients Scheduled* $_{n}$} & & \multicolumn{2}{c}{ Fraction of Patients Arrived $^{\dagger}$} \\
\cline { 2 - 5 } Days from Referral & Before the Intervention & $\begin{array}{c}\text { After the } \\
\text { Intervention }\end{array}$ & & $\begin{array}{c}\text { Before the } \\
\text { Intervention }\end{array}$ & $\begin{array}{c}\text { After the } \\
\text { Intervention }\end{array}$ \\
\hline 30 & 0.39 & 0.63 & 0.29 & 0.42 \\
60 & 0.52 & 0.70 & 0.39 & 0.49 \\
90 & 0.58 & 0.72 & 0.44 & 0.52 \\
\hline
\end{tabular}

${ }^{*} P<.001, \log$ rank test.

${ }^{\dagger} P=.001, \log$ rank test.

above (hazard ratio, $1.35 ; 95 \%$ confidence interval, 1.13-1.62). The fraction of patients who successfully scheduled $\mathrm{BH}$ visits increased at all time points after referral in the postintervention period compared with the period before the intervention $(P<.001)$. A similar effect was observed for the fraction of patients arriving for care $(P=.001)$. This was apparent for all time periods through 90 days (Table 5).

\section{Survey Results}

Survey response rates were $40 \%$ before implementation and $40 \%$ afterward. In the domain "Acceptance of Lean as a QI Method," the median score increased from 4.0 to $6.0(P=.35)$ for 1 statement ("The QI study was easy to do") and was unchanged at 6.0 for the other ("If I were asked to be part of a QI project in the future, I would accept") $(P=.95)$. The median scores increased for both statements in the domain "Workflow Effectiveness": the first ("The QI study made my job easier") from 5.0 to $7.0(P=.11)$, and the second ("The QI project made us more efficient as a practice") from 5 to $6(P=.61)$. The median score for the domain of "Clinical Effectiveness" (for the statement "The QI project was successful in improving the quality of patient care") increased from 5.5 to $6.0(P=.77)$.

\section{Feedback from Participants}

We presented the results of the EHR data and survey responses to study participants 10 months after the implementation of integrated $\mathrm{BH}$ services. Providers and staff responded with agreement on the improved clinical results of the project, its positive effect on workflow, and the usability of Lean as a QI method. The following are sample comments written by participants or recorded during the presentation:
- "It was the 'mapping' process [system diagrams] that worked so well. ... If you can see it, you can understand it." (medical office assistant)

- "You do not see what is happening to each other when you are working-we're so wrapped up in our own work. We could see this." (advanced practice nurse)

- "I can tell this is happening, that it is working, just by the calls I get now. Patients are getting the care they need." (triage nurse)

- "It made sense out of chaos." (provider)

\section{Discussion}

Office practices typically have limited time to invest in complex change. An effective QI method in health care is characterized by 2 key strategies: directly engaging front-line workers ${ }^{25}$ and eliminating wasteful work processes. ${ }^{26}$ As suggested by Fischer et $\mathrm{al}^{27}$ over 15 years ago, and illustrated by $\operatorname{Sinksy}^{28}$ in a provider office practice, health care workers are able to efficiently and successfully redesign key office systems and processes.

The value of Lean lies in its systematic ability to address clinical and operational requirements while improving patient care. With the advance of health care reform confronting health care providers and leaders with a constant flow of change, a model for implementing effective, efficient, and acceptable change methods is of value. The use of Lean to integrate a $\mathrm{BH}$ clinician and change the $\mathrm{BH}$ referral process showed significant improvements in care, especially in treatment initiation. As a QI process, Lean had strengths in its focus on improving work processes, system perspective, and success in achieving the team's objective. Although this application of Lean was limited to 1 primary care setting 
and intervention, the documented application of Lean in a range of health care systems and change efforts ${ }^{16,17,23,25}$ indicates that it is likely to have broad application in practice sites.

\section{Limitations}

As a single case study, generalizability to other clinical practices is not expected. Providers and staff on the QI team were not blinded to the purpose of this study and were likely to have developed an attachment to the results of their own work, biasing some survey results and possibly affecting the responses of other clinic providers and staff. It is unlikely that team members' opinions affected the data collected by the EHR system regarding scheduling, treatment, and the days elapsed since the referral date. Although survey responses tended to show improvement after the intervention, the sample was too small to achieve statistical significance.

\section{Conclusions}

The discovery of a better model of care (in this case, integrating $\mathrm{BH}$ in primary care) calls for an effective method of implementing it. Using Lean, this $\mathrm{BH}$ implementation project was associated with significant improvements in most measures of performance. The Lean process itself was brief (8 hours), adaptable to an important and complex intervention (BH integration), improved workflow design (eg, direct scheduling of $\mathrm{BH}$ appointments), provided a system perspective ("it was the mapping process"), and achieved the team's objectives (improved treatment initiation). Lean is a promising implementation strategy that may offer tangible benefits and find acceptance among providers and staff. It warrants more extensive evaluation in a variety of settings.

\section{References}

1. Kathol RG, Degruy F, Rollman BL. Value-based financially sustainable behavioral health components in patient-centered medical homes. Ann Fam Med 2014;12:172-5.

2. Kessler LG, Cleary PD, Burke JD Jr. Psychiatric disorders in primary care. Results of a follow-up study. Arch Gen Psychiatry 1985;42:583-7.

3. Mental disorders in general medical practice an opportunity to add value to healthcare. Behav Healthc Tomorrow 1996;5:55-62, 72.

4. Katon W. The epidemiology of depression in medical care. Int J Psychiatry Med 1987;17:93-112.
5. Kessler R, Stafford D. Primary care is the de facto mental health system. In: Kessler R, Stafford D, eds. Collaborative medicine case studies: evidence in practice. New York: Springer; 2008: $9-21$.

6. Cunningham PJ. Beyond parity: primary care physicians' perspectives on access to mental health care. Health Aff (Millwood) 2009;28:w490-501.

7. Kessler R. Mental health care treatment initiation when mental health services are incorporated into primary care practice. J Am Board Fam Med 2012;25:255-9.

8. Baird M, Blount A, Brungardt S, et al. Joint principles: integrating behavioral health care into the patient-centered medical home. Ann Fam Med 2014; 12:184-5.

9. Kessler R, Miller BF, Kelly M, et al. Mental health, substance abuse, and health behavior services in patient-centered medical homes. J Am Board Fam Med 2014;27:637-44.

10. Hunter CL, Goodie JL. Operational and clinical components for integrated-collaborative behavioral healthcare in the patient-centered medical home. Fam Syst Health 2010;28:308-21.

11. Hunter C, Goodie J, Oordt MS, Dobmeyer AC. Integrated behavioral health in primary care: stepby-step guidance for assessment and intervention. Washington, DC: American Psychological Association; 2009.

12. Butler M, Kane RL, McAlpine D, et al. Integration of mental health/substance abuse and primary care. Evid Rep Technol Assess (Full Rep) 2008;(173):1-362.

13. Kessler R. An outcome and clinical research focus in an integrated care patient-centered medical home. In: Curtis R, Christian E, ed. Integrated care: applying theory to practice. Oxford, UK: Routledge; 2012; 297-310.

14. Jimmerson C, Weber D, Sobek DK 2nd. Reducing waste and errors: piloting lean principles at Intermountain Healthcare. Jt Comm J Qual Patient Saf 2005;31:249-57.

15. Womack JP, Jones DT. Lean thinking: banish waste and create wealth in your corporation. New York: Simon \& Schuster; 1996.

16. Gamm L, Kash B, Bolin J. Organizational technologies for transforming care: measures and strategies for pursuit of IOM quality aims. J Ambul Care Manage 2007;30:291-301.

17. Kenney C. Transforming health care: Virginia Mason Medical Center's pursuit of the perfect patient experience. New York: Productivity Press; 2011.

18. van Eeghen C. Improving the quality of quality improvement: case studies of provider office practices in the use of assisted office systems analysis within a quality improvement project. Chapel Hill, NC: Health Management and Policy, University of North Carolina; 2012.

19. Kessler R. Identifying and screening for psychological and comorbid medical and psychological 
disorders in medical settings. J Clin Psychol 2009; 65:253-67.

20. Bate P, Mendel P, Robert G. Organizing for quality: the improvement journeys of leading hospitals in Europe and the United States. Oxon, UK: Radcliffe Publishing; 2008.

21. Young J, Gilwee J, Holman M, Messier R, Kelly M, Kessler R. Mental health, substance abuse, and health behavior intervention as part of the patientcentered medical home: a case study. Transl Behav Med 2012;2:345-54.

22. Auxier A, Runyan C, Mullin D, Mendenhall T, Young J, Kessler R. Behavioral health referrals and treatment initiation rates in integrated primary care: a Collaborative Care Research Network study. Transl Behav Med 2012;2:337-44.
23. Zidel TG. A Lean guide to transforming healthcare. Milwaukee, WI: American Society for Quality, Quality Press; 2006.

24. Davis FD. Perceived usefulness, perceived ease of use, and user acceptance of information technology. MIS Q 1989;13:319-40.

25. Young T, Brailsford S, Connell C, Davies R, Harper $\mathrm{P}$, Klein JH. Using industrial processes to improve patient care. BMJ 2004;328:162-4.

26. Boat TF, Chao SM, O’Neill PH. From waste to value in health care. JAMA 2008;299:568-71.

27. Fischer LR, Solberg LI, Kottke TE. Quality improvement in primary care clinics. Jt Comm J Qual Improv 1998;24:361-70.

28. Sinsky CA. Improving office practice: working smarter, not harder. Fam Pract Manag 2006;13:28-34. 